\title{
Das Prinzip demokratischer Gleichheit
}

\author{
Kippfiguren als Gedankenexperimente
}

\section{The principle of democratic equality \\ Reversible figures as thought experiments}

\section{Christine Abbt, Graz}

Zusammenfassung: Kippfiguren, so die These im Beitrag, sind Gedankenexperimente, mit denen das demokratische Prinzip Gleichheit, und also das darin unauflöslich wirksame und paradoxe Verhältnis von Gleichheit und Freiheit, plausibel wird. Das geschieht auf zwei Ebenen. Im Vollzug des Kippens wird das je eigene Sehen als Perspektive kenntlich und eine Differenzierung zwischen Wahrnehmen und Denken kommt in Gang. Zudem wird erkennbar, dass dasselbe gleichzeitig dasselbe und doch nicht dasselbe ist. Paradoxe Verhältnisse werden so dem Denken zugänglich und Verbindendes vergegenwärtigt. Das Denkerlebnis, das an und mit etwa der Kippfigur des Enten-Hase-Kopfs mittelbar wird, reduziert zwar die Komplexität der Thematik auf eine überschaubare Dimension, es bereitet dabei allerdings doch darauf vor, Anderen als Freie und Verschiedene und als Gleiche zu begegnen. Bewusstwerdung und Rationalität führen, so wird mit dem Gedankenexperiment der Kippfiguren deutlich, nicht, wie es etwa Jean-Jacques Rousseau in seiner Abhandlung über die Ungleichheit unter den Menschen und der darin formulierten Forderung nach Gleichheit ausführte, weg von der Einsicht in die Gleichheitsidee, sondern verhelfen dieser hier erst zur Anerkennung und Durchsetzung.

Schlagwörter: Kippfiguren, Gedankenexperiment, Gleichheit, Differenz, Freiheit, Demokratie, Anthropologie, Performativität, Wittgenstein, Kant

Abstract: Reversible figures, so the thesis in the article, are thought experiments with which the democratic principle of equality, and thus the inextricably effective and paradoxical relationship between equality and freedom, becomes plausible. This happens on two levels. In the process of tilting, one's own seeing becomes recognizable 
as a perspective and a differentiation between perceiving and thinking is initiated. In addition, it becomes recognizable that the same thing is simultaneously the same and yet not the same. Paradoxical relationships thus become accessible to thinking, and what unites all people is brought to mind. The experience of thinking, which becomes indirect with the tilting figure of the duck-hare head, reduces the complexity of the topic to a manageable dimension, but it prepares us to encounter others as free and different and as equals. Deeper thought, awareness and rationality do not lead away from the insight into the idea of equality, as, for example, Jean-Jacques Rousseau explained in his treatise on inequality among men and the demand for equality formulated therein, but rather help this idea to be recognized and asserted.

Keywords: Reversible figure, thought experiment, equality, difference, freedom, democracy, anthropology, performativity, Wittgenstein, Kant

\section{Inwiefern handelt es sich bei Kippfiguren um Gedankenexperimente?}

Wenn in der Philosophie von Gedankenexperimenten die Rede ist, wird selten auf Kippfiguren verwiesen. Tatsächlich handelt es sich bei Kippfiguren nicht um imaginative oder fiktive Gedankenexperimente etwa im Sinne des Schleiers des Nicht-Wissens von John Rawls oder dem erkrankten Geiger von Judith Jarvis Thomson. Kippfiguren sind dennoch als Gedankenexperimente zu bestimmen. Sie entsprechen allen zentralen Kriterien, welche in der jüngeren Literatur über Gedankenexperimente angeführt werden. Gedankenexperimente werden dort erstens als Hilfsmittel ausgewiesen, mit denen Theorien weiterentwickelt, untermauert, anschaulich gemacht oder widerlegt werden können. Den Gedankenexperimenten wird dabei zweitens eine spezifische Plausibilisierungsqualität zugesprochen. Sie vermitteln auf unerwartete Weise und oftmals über kognitiv leicht zugänglichem (Um-) Weg Einsicht in anspruchsvolle Zusammenhänge, eröffnen neue Perspektiven und entlarven Fehlannahmen. Gedankenexperimente verlangen zudem drittens eine rationale Vertiefung und Integration oder Übersetzung des anschaulich Gewordenen auf einen weiteren Kontext. ${ }^{1}$ Diese Bestimmungen treffen auf Kippfiguren zu: Ausgehend von Kippfiguren lassen sich Theorien

$1 \quad$ Wolfgang Buschlinger: Denk-Kapriolen? Gedankenexperimente in Naturwissenschaften, Ethik und Philosophy of Mind, Würzburg 1993; Johannes Rohbeck (Hg.): Anschauliches Denken, Dresden 2005; Martina Peters, Jörg Peters: Philosophieren mit Gedankenexperimenten, Band I und II, Hamburg 2020. 
entwickeln, anschaulich machen, untermauern oder widerlegen. Die im Umgang mit Kippfiguren realisierte Einsicht ist sodann kognitiv leicht zugänglich und hat eine hohe und spezifische Plausibilisierungsqualität. Erst die rationale Vertiefung schließlich legt deren weiteres philosophisches und, wie hier ausgeführt wird, demokratiefreundliches Potential frei. Kippfiguren ermöglichen eine Einsicht, die nicht allein auf die Wahrnehmung der Figur zurückgeht, sondern sich erst im Zusammenspiel zwischen Erleben und Denken und in der sich daran anschließenden Reflexion und Schlussfolgerung vollständig durchsetzt.

Kippfiguren sind Experimente der Wahrnehmung und des Denkens. Sie bieten zwei oder mehrere Perspektiven an, die auf eine einzige Raumstelle reduziert sind, und überraschen so mit Entdeckungen und ermöglichen Einsichten. Das gelingt bei Kippfiguren, indem dasselbe sich ausschließlich unterschiedlich zeigt und sich dabei in der Person, welche die Alternativen vollzieht, nicht nur ein Erleben, sondern damit verbunden ein Denkprozess realisiert, der das Wahrgenommene interpretieren und aus dem Gesehenen Schlüsse ziehen lässt, die ihrerseits aus plausiblem Grund zu weiteren Verallgemeinerungen Anlass bieten. Bei der bekannten Hasen-Enten-Kopf-Kippfigur etwa bedeutet dies, dass dasselbe einmal als Hase und einmal als Ente sichtbar zu machen ist. Die Kippfigur lässt spielerisch evident und bewusstwerden, dass es Alternativen zur eigenen ersten Wahrnehmung gibt und in einem zweiten Schritt zudem, dass diese Alternativen weder sofort noch gleichzeitig in den Blick kommen können. Beide Aktualisierungen, also Hase und Ente gleichzeitig in den Blick zu nehmen, gelingt ebenso wenig wie den Moment des Kippens sinnlich festzuhalten. Kippfiguren lassen darüber hinaus begreifen, dass das Verbindende und Gemeinsame nicht zwingend wahrgenommen, aber trotzdem abgeleitet und denkend anerkannt werden kann, ja sogar aus sinnvollem Grund anerkannt werden muss. In der rationalen Vertiefung werden so Verschiedenheit und Gleichheit als gleichzeitige Möglichkeit desselben verständlich und zudem wird ein Sensorium für die Differenzierung zwischen Wahrnehmen und Denken etabliert.

Ludwig Wittgenstein verdeutlichte seinerseits, inwiefern es sich bei Kippfiguren um wichtige Gedankenexperimente handelt und deutete an, worin eine Bedeutung von Kippfiguren für Fragen und Anliegen der praktischen Philosophie liegen könnte. Ausgehend von Wittgensteins Überlegungen zu reversiblen Figuren und seiner Gewichtung des Kippmoments, in dem sich Erleben und Denken eng aufeinander beziehen, wird im Folgenden nach der besonderen Bedeutung von Kippfiguren für die Plausibilisierung des demo- 
kratischen Prinzips der Gleichheit aller Menschen gefragt und dargelegt, wie mit Kippfiguren das paradoxale Einhergehen von Gleichheit und Differenz einsichtig und das eigene Sehen als Perspektive erkennbar wird.

\section{Wittgensteins Antwort und Begründung}

Wie ist es möglich, dass wir ein und dieselbe unveränderte Figur einmal als das Eine und ein anderes Mal als etwas Anderes sehen können? Sehen wir die Figur je als etwas Verschiedenes oder deuten wir nur unterschiedlich? „Ich will fragen:“ - heißt es in Wittgensteins Bemerkungen über die Philosophie der Psychologie - „worin besteht es, die Figur einmal so, einmal anders sehen? - Sehe ich wirklich jedesmal etwas anderes oder deute ich nur, was ich sehe, auf verschiedene Weise?“2 Wittgenstein bedient sich unterschiedlicher Figuren und Bilder, an denen er seine Überlegungen entwickelt, etwa das Quadrat mit den Diagonalen, das auch als Pyramide gesehen werden kann oder das Drahtgestell, das auch einen Glaswürfel darstellt, oder eben die Figur, die sich einmal als Hasen- und einmal als Entenkopf aktualisieren lässt. Wittgensteins Interesse gilt dabei zunächst der Frage nach dem Verhältnis von Sehen und Deuten. Je später die Überlegungen im Gesamtwerk angesiedelt sind, desto mehr Aufmerksamkeit wird dem Kippen bzw. dem sich dabei ereignenden Denkerlebnis gewidmet.

In Bezug auf die reversiblen Figuren entwickelt Wittgenstein den Begriff des ,Sehen als‘. Denn die spezifische Weise von Sehen, welche im Umgang mit Kippfiguren offensichtlich wird, ist weder mit Sehen noch mit Deuten gleichzusetzen. Sie ist zwar begrifflich verwandt mit dem Sehen, aber nicht identisch. Sie funktioniert zwar nicht getrennt von Deuten, aber sie fällt auch nicht damit zusammen. Wittgenstein konstruiert begrifflich eine Art des Sehens, die zwischen Wahrnehmen und Denken zu verorten ist. ${ }^{3}$ In den Vorstudien zu den Philosophischen Untersuchungen ist dabei von der

2 Ludwig Wittgenstein, (1946/47): Bemerkungen über die Philosophie der Psychologie. In: ders.: Werkausgabe, Bd. 7., Frankfurt a. M. 1984, 8.

$3 \quad$ Seeing-as can be located between seeing (pure perception) and thinking (pure interpretation), but it is in some cases (like in the case of the dubbit) closer to the interpretation.“ Kristijan Krkač: „Wittgenstein's Dubbit III. Caught in Kirchberg am Aspekt-Wechsel“. In: Wittgenstein-Studien, 1/2010, Berlin/ New York, 121-150, 143. 
besonderen und bedeutsamen Qualität dieses Sehens die Rede, für die fortan die Bezeichnung ,Sehen als' oder ,Aspektsehen' verwendet wird.

Zur Veranschaulichung dessen, was als ,Sehen als' oder als ,Aspektsehen' zu gelten hat, wird von Wittgenstein folgendes Beispiel angeführt: „Ich mag zwei Gesichter, die sich nicht ändern, betrachten: auf einmal leuchtet eine Ähnlichkeit in ihnen auf. Ich nenne diese Erfahrung das Aufleuchten eines Aspekts. “" Zuerst werden zwei Gesichter gesehen, dann wird eine Ähnlichkeit vergegenwärtigt. Die neue Sichtweise stellt sich „auf einmal“ ein, sie kommt neu hinzu, obgleich immer noch gesehen werden kann, dass sich die Gesichter nicht verändert haben. In Wittgensteins Beispiel werden die drei Kategorien des ,Sehens', des ,Sehens als' und des ,Deutens ' zunächst so auseinandergehalten, wie es sich in der praktischen Erfahrung kaum verhält. Gemäß Wittgenstein werden demnach zuerst die Gesichter gesehen, dann wird ein neuer Aspekt bemerkt und dann kann festgestellt werden, dass sich zwar die Gesichter nicht verändert haben, dass es aber eine Veränderung gegeben hat. Wahrnehmen und Denken finden in dieser Beschreibung in einer zeitlichen Abfolge nacheinander statt. Die eindeutige Kategorisierung in ,Sehen', in ,Sehen als" und in ,Deuten ' wird im Verlauf der Überlegungen allerdings auch von Wittgenstein problematisiert und zurückgewiesen. Ist das Sehen des Gesichts nicht auch schon ein ,Sehen als'? Gibt es ein Sehen, welches nicht als ein ,Sehen als zu qualifizieren wäre? Dem Einwand, dass es sich beim Sehen immer schon um ein ,Sehen als etwas' handelt, wird Recht gegeben. Zwar werden ,Sehen als' und ,Denken' von Wittgenstein weiterhin nicht gleichgesetzt. Der Übergang zwischen ,Sehen als` und ,Deuten` wird aber als grundlegender Bestandteil nicht nur der Wahrnehmung, sondern vor allem auch der gedanklichen Vertiefung vorgestellt. ${ }^{5}$ Deutung wäre anders kaum sinnvoll zu denken, wenn nicht durch eine stets neu angesetzte Bezugnahme auf Unverändertes. Der Fokus verschiebt sich für Wittgenstein in der Folge: Er ist fortan nicht mehr auf das Sehen oder das Sehen eines Aspekts gelegt und auch nicht mehr auf die Beziehung, die zwischen Betrachter:in und Betrachtetem besteht, sondern der Fokus liegt jetzt auf dem

4 Ludwig Wittgenstein (1948/49): „Vorstudien zum zweiten Teil der Philosophischen Untersuchungen“. In: ders.: Werkausgabe. Bd. 7. Frankfurt a. M.1984, 408.

$5 \quad$ Zum Zusammenhang zwischen Aspektwechsel-Erlebnis und Denken: Thorsten Jantschek: „Wittgenstein über Sehen und Sehen-als“. In: Wittgenstein Studies 2/96. Bristol 1996. 
Moment des Kippens. Die spezifische Plausibilisierungsqualität von Kippfiguren liegt in diesem Umschalten begründet, in dem sich ,Sehen als ein ,Sehen als ${ }^{c}$ konkretisiert und zeigt. Wittgenstein bestimmt diesen Kippmoment als „Aspektwechsel“ und schreibt ihm zentrale Bedeutung zu. Es handelt sich dabei um jenen entscheidenden Moment, in dem jemand sein eigenes Sehen als Perspektive vergegenwärtigen kann.

\section{Die eigene Sicht als Perspektive}

Die Kippfigur wird zum Gedankenexperiment, sobald sich in Anbetracht einer gleichbleibenden Figur die Einsicht etabliert, dass daran noch etwas Zweites und Weiteres zu vergegenwärtigen ist. Voraussetzung dafür ist jenes Kippen, das sich in der Formulierung ausdrückt: „Jetzt sehe ich es nicht mehr als Ente, sondern jetzt sehe ich es als Hase!“ Erst in einem nächsten Schritt kann davon ausgehend festgehalten werden, dass dieselbe Figur sowohl als Ente als auch als Hase gesehen werden kann und dass es sich hierbei bemerkenswerterweise um zwei verschiedene und dabei gleichrangige Perspektiven auf ein und dasselbe handelt.

„Im Aspektwechsel wird man sich des Aspekts bewusst“, heißt es bei Wittgenstein in den Vorstudien zum zweiten Teil der Philosophischen Bemerkungen. ${ }^{6}$ Der Aspektwechsel wird als Voraussetzung dafür erläutert, dass ein Aspekt überhaupt als ein Aspekt vergegenwärtigt werden kann. Oder anders gesagt: erst durch den Vollzug eines Aspektwechsels wird das eigene Sehen als Perspektive kenntlich. Wenn jemand neben dem Hasenkopf auch jenen der Ente entdeckt, wird nicht nur ein neues Bild generiert, sondern gleichzeitig auch eine neue Sicht auf das eigene Sehen. Das Selbstverständliche wird durch die neue Sehweise problematisiert und als relativ und möglich statt als vollständig und notwendig ausgewiesen.

Interessant ist, dass der Vollzug des Aspektwechsels kaum je frustriert, sondern meistens zu einem regen Hin-und-Her-Kippen zwischen den möglichen Aktualisierungsvarianten verleitet und dabei erfreut. Das Bewusstwerden der Alternative und die damit verbundene Relativierung der Absolutheit des eigenen ersten Sehens erhellt, so scheint es, Gemüt und Geist. Wittgenstein bestimmt den Aspektwechsel als ein grundlegendes Denkerlebnis. Mit Fokus auf die zeitliche Abfolge des gedanklichen Prozes-

6 Ludwig Wittgenstein (1948/49): „Vorstudien zum zweiten Teil der Philosophischen Untersuchungen“. In: ders.: Werkausgabe. Bd. 7, Frankfurt a. M.1984, 375 . 
ses kann der Aspektwechsel auch als Erleben-Denken beschrieben werden. Dieses Erleben-Denken bildet den Angelpunkt für ein exaktes Verständnis des Verhältnisses von Sehen und Deuten und darüber hinaus hat es eine grundlegende Bedeutung in Bezug auf das Verstehen der Person selbst, die einen Aspektwechsel vollzieht. Mit und an Kippfiguren erschließt sich einem eine neue Sicht auf das eigene Sehen. Das Sehen zeigt sich. Es tritt aus der Gewohnheit und Selbstverständlichkeit heraus und erhält ein Profil. ${ }^{7}$

Damit der Aspektwechsel allerdings seine Wirkung entfalten kann, muss er je konkret von einer Person realisiert werden. Diese Bedingung erscheint offensichtlich. Sie ist aber zentral und gilt auch im Zusammenhang mit anderen Gedankenexperimenten. Der individuelle (Nach-)Vollzug des Experiments bildet eine Voraussetzung für die Durchsetzung der erwähnten spezifischen Plausibilisierungsqualität, die sich eben aus dem Zusammenspiel von Erleben und rationaler Vertiefung ergibt. Wittgenstein verweist seinerseits auf den Unterschied, der zwischen dem Wissen über das Kippen und dem Vollzug des Kippens bei Kippfiguren besteht. Jemandem nur von einer Figur zu erzählen, die einmal als eine Ente und einmal als ein Hase gesehen werden könne, würde kaum in demselben Maß faszinieren und transformieren wie es der Vollzug des Kippmoments vermag. ${ }^{8}$ Auch darum beschreibt Wittgenstein das Ausbleiben solchen Vollzugs und der damit verbundenen Einsichten als höchst problematisch. Personen, die nicht fähig sind, an einer Kippfigur zwei Aktualisierungen vorzunehmen, nennt Wittgenstein „aspektblind“. Eine aspektblinde Person hat nicht das Erlebnis des Aufleuchtens eines Aspekts. Sie „(...) wird zu Bildern überhaupt ein anderes Verhältnis haben als wir." "In ihrem Sprachgebrauch würde die Person kein Gespür für die Eigentümlichkeit der Worte haben; ihr würde die „Anhänglichkeit“ ${ }^{10}$ an

$7 \quad$ „Der Aspektwechsel als Er-Eignis ist ein [...] faktisches Sich-Zeigen.“ Chris Bezzel: „Philosophie dürfte man eigentlich nur dichten. Über Ludwig Wittgenstein“. In: Richard Faber/Barbara Naumann (Hg.): Literarische Philosophie, philosophische Literatur, Würzburg 1999, 153-169, 165.

8 Konsequenterweise bildet Wittgenstein die Figuren, die er thematisiert, stets $\mathrm{ab}$.

9 Ludwig Wittgenstein (1948/49): „Vorstudien zum zweiten Teil der Philosophischen Untersuchungen“. In: ders.: Werkausgabe. Bd. 7, Frankfurt a. M.1984, 449.

10 Gunter Gebauer: Wittgensteins anthropologisches Denken, Beck'sche Reihe 1886, München 2009, $215 f$. 
ihre Worte“ fehlen. „Man sagt, Einer habe kein ,musikalisches Gehör“, und ,Aspektblindheit“ ist (etwa) mit dieser Art Gehörlosigkeit zu vergleichen." ${ }^{11}$

Wittgenstein spricht dem Vermögen zu Aspektwechseln elementare Bedeutungen zu und zwar, wie schon erwähnt, einerseits für die Klärung des Verhältnisses von Sehen und Deuten und andererseits auch hinsichtlich der Herausbildung einer persönlichen Haltung. Wittgenstein verwendet zur Beschreibung des Ereignisses im Aspektwechsel Begriffe wie „aufleuchten“, „erscheinen“, „,aufflackern“, „fluktuieren“, „verwandeln“, „umschlagen“. Das „Aufleuchten des Aspekts“, und das ist hier in Bezug auf die Kippfiguren als Gedankenexperimente wichtig, etabliert in der betrachtenden Person eine veränderte Einstellung. ${ }^{12}$ Durch das Sehen der Alternative und im Bemerken des eigenen Sehens als Perspektive wird ein Verstehen möglich, das auch ein Sich-Verstehen umfasst und zu Unterscheidungen zwischen wahr-nehmen und wahr-denken veranlasst. ${ }^{13}$ Das Ausbleiben des Aspektwechsels führte nicht nur zu einem anderen, sondern zu einem defizitären Verhältnis zu Bildern und Tönen, zur Sprache und gegenüber sich selbst. Der Aspektblinde hätte nicht nur keine Anhänglichkeit an die eigenen Worte, wie Wittgenstein schreibt. Er hätte auch keine Möglichkeit, diese Anhänglichkeit als Anhänglichkeit, als Vorliebe durch Gewöhnung zu bemerken und damit keine Chance, zwischen notwendig und möglich oder zwischen Vorurteil und Urteil, zwischen Wahrnehmen und Deuten zu differenzieren. Faszination, Überzeugungskraft und Bedeutung der Kippfiguren liegen in ihrer Eigenschaft, dem Einzelnen ein Erleben-Denken zu ermöglichen und aufzuzwingen, welches das eigene Denken in Gang bringt, es in ein Selbst-Verhältnis setzt, herausfordert und fördert. Das Erleben-Denken mit Kippfiguren ist in diesem Sinne als transformativ zu bezeichnen.

11 Ludwig Wittgenstein (1948/49): „Vorstudien zum zweiten Teil der Philosophischen Untersuchungen“. In: ders.: Werkausgabe. Bd. 7, Frankfurt a. M.1984, 450 .

„The organisation of a drawing of the dubbit, or organisation of our seeing, is not changed (against behaviourism and Gestalt psychology), rather our attitude is changed." Hans-Johann Glock: A Wittgenstein Dictionary, Oxford 1996, 39 .

„Das Auffallen ist dem Denken verwandt.“ Ludwig Wittgenstein (1948/49): „Vorstudien zum zweiten Teil der Philosophischen Untersuchungen“. In: ders.: Werkausgabe. Bd. 7, Frankfurt a.M.1984, 441. 


\section{Dasselbe und doch nicht dasselbe}

Kippfiguren laden ein zum spielerischen Umgang und strukturieren dabei gleichzeitig einen bestimmten Prozess von Erleben-Denken. Wenn eine zweite Aktualisierung gelingt und neben der Ente auch der Hase als gleichrangige Alternative ersichtlich ist, dann wird die eigene Wahrnehmung als Perspektive augenfällig. Damit hört das Experiment in seiner Wirkkraft und Aussage aber noch nicht auf. Das Staunen über die mögliche Alternative und das Bemerken des eigenen selektiven Zugangs wirft weitere Fragen auf. Wittgenstein hält fest: „Das Seltsame ist eigentlich das Staunen; das Fragen ,Wie ist es möglich!' Der Ausdruck davon ist etwa: Dasselbe - und doch nicht dasselbe." ${ }^{14}$

Wenn das Kippen gelingt, wird eine Alternative zur ersten Aktualisierung ersichtlich. Im Hin- und Her-Kippen findet eine Vergewisserung statt: Es sind zwei voneinander unabhängige Aktualisierungen möglich, nämlich eben etwa Ente und Hase. ${ }^{15}$ Wer das Kippen beherrscht und die Ente und auch den Hasen nacheinander aufscheinen lassen kann, realisiert allerdings bald, dass es nicht gelingt, Ente und Hase gleichzeitig zu visualisieren. Beide Aktualisierungen gleichzeitig zu vollziehen, ist nicht möglich. Entweder man sieht die Ente oder den Hasen. ${ }^{16}$

Kippfiguren lassen sich im besten Falle also sinnlich als eine oder zwei (oder mehrere) Figuren sehen. Sie verschließen sich hingegen gegenüber der Möglichkeit einer gleichzeitigen Aktualisierung von Verschiedenem. Nacheinander sind Ente oder Hase zu sehen, aber nicht gleichzeitig beides zusammen. Dass Verschiedenes auf dasselbe zurückzuführen ist, und weiter noch, dasselbe ist, entspricht einem Schluss, der erst durch den rationalen

14 Ludwig Wittgenstein (1948/49): „Vorstudien zum zweiten Teil der Philosophischen Untersuchungen“. In: ders.: Werkausgabe. Bd. 7. Frankfurt a. M.1984, 376 .

15 Zu den Prozessen und Wirkungen der Wiederholung vgl.: Svenja Flaßpöhler, Tobias Rausch, Tina Wald (Hg.): Kippfiguren der Wiederholung. Interdisziplinäre Untersuchungen zur Wiederholung in Literatur, Kunst und Wissenschaft, Frankfurt am Main 2007.

16 Über die Unmöglichkeit, den Moment des Übergangs festzuhalten, gehen weiter ein: Christiane Thompson, Gabriele Weiß, Oliver Krüger: Die Grenze des Verstehens. Überlegungen zum Verhältnis von Ironie und Pädagogik, in: Bildende Widerstände - widerständige Bildung: Blickwechsel zwischen Pädagogik und Philosophie, Bielefeld 2015, 61-78. 
Nachvollzug des Wahrgenommenen gezogen werden kann und der vom Erleben-Denken zwar abhängt, aber selbst nicht allein sinnlich einzuholen ist. Die Übersetzung der Entweder-oder-Relation bei Kippfiguren in eine Sowohl-als-auch-Struktur nimmt ihren Ausgangspunkt im Kippmoment und konkretisiert sich nachträglich durch eine gedankliche Vertiefung. Verbindendes und Allgemeines werden an und mit Kippfiguren nicht sichtbar, aber in der reflektierenden Analyse verständlich.

Das paradoxale Phänomen ist nicht unmittelbar sinnlich einzufangen, sondern erst und hier nur rational. Zu sehen sind Ente oder Hase. Da beides nacheinander sichtbar ist und weil gleichzeitig festgestellt werden kann, dass die Figur bzw. die Linie sich durch alle Aktualisierungen hindurch nicht und nie verändert, drängt sich der Schluss zwingend auf, dass dasselbe als Verschiedenes betrachtet werden kann, auch wenn es sich nicht ändert. Die Gleichheit wird durch die eine oder andere Aktualisierung ausgeblendet, aber es ist plausibel, eine solche anzunehmen und rational beurteilt, muss sogar von der Gleichheit ausgegangen werden.

\section{Kippfiguren und das Prinzip demokratischer Gleichheit}

Kippfiguren machen zwei unverzichtbare Grundlagen des demokratischen Horizonts zum Diktat: a) ,Sehen bedeutet ,Sehen als' (Perspektivität) und b) dasselbe ist dasselbe und doch nicht dasselbe (Paradoxon von Gleichheit und Freiheit aller Menschen).

a) Erst wenn die Prozesse der fortlaufenden Totalisierung der Wahrnehmung unterbrochen werden, kann sich ein Bewusstsein für die je eigene Größe und Relativität entwickeln. Die sinnliche Wahrnehmung kann eine totalisierende Wirkung entfalten, indem sie der betrachtenden Person eine Ausschließlichkeit vermittelt, welche irreführend ist. Durch das Bemerken einer gleichrangigen Alternative im Erleben-Denken des Kippmoments wird Sehen als Perspektive kenntlich und für eine allgemeine Problematisierung zugänglich. Wenn ich nur den Hasen sehe, was übersehe ich sonst noch alles (zumindest auf den ersten Blick)? Die Anerkennung der eigenen Wahrnehmung als eine fragile Perspektive unter anderen gleichberechtigten und die Schlussfolgerung aus der rationalen Vertiefung des Erleben-Denkens, dass es nicht nur an und mit Kippfiguren Perspektiven gibt, sondern ebenso in anderen Konstellationen und Kontexten und dass davon abgeleitet zunächst einmal allgemein von Perspektivität ausgegangen werden muss, leitet über zu einer für Demokratien zentralen Anerkennung von Perspektivität des Zu- 
griffs und der Aufwertung des Möglichen vor dem Notwendigen. Dasselbe kann mehr oder anderes sein als zuerst angenommen und Gleiches kann unterschiedlich gesehen werden oder Verschiedenes sein.

Der Zusammenhang zwischen der Anerkennung des eigenen Sehens als einer Perspektive unter anderen und der Anerkennung demokratischer Gleichheit ist in der Literatur vielfach behandelt und unterstrichen. ${ }^{17}$ Die Einsicht, dass der eigene Blick überraschend deutlich verstellt sein und dasselbe als Verschiedenes aktualisiert werden kann, erscheint dabei grundlegend etwa für die Bereitschaft, die eigene Wahrnehmung durch jene der Anderen in Frage stellen zu lassen und für die Bereitschaft, gemeinsam fortlaufend zu prüfen, welche der Sehweisen sich als sinnvoll, begründet und wünschenswert erweisen kann und ob es weitere, noch nicht gesehene oder noch nicht einbezogene gibt. ${ }^{18}$ Die Frage drängt sich daher noch einmal hinsichtlich derjenigen auf, die dazu nicht bereit oder nicht in der Lage sind. Bei Immanuel Kant werden solche Personen als Egoisten beschrieben und mit einem Zyklopen verglichen, dem ein zweites Auge fehlt. Ebenso wie Wittgenstein spricht auch Kant der Einsicht in die defizitäre eigene Wahrnehmung grundlegende Bedeutung zu und zwar für alle Erkenntnis, für Ethik und Ästhetik und für das Zusammenleben von Menschen, die sich an den Prinzipien von Selbstbestimmung und Gleichheit orientieren. ${ }^{19}$ Kant verweist in seinen Ausführungen, wie erwähnt, diesbezüglich auf den Vergleich des Menschen mit einem Zyklopen. Wer nur mit einem Auge sieht, der kann sich im übertragenen Sinn nicht als ein Weltenbürger unter anderen verstehen, so Kant. Wittgenstein bezeichnet dasselbe Defizit als „Aspektblindheit“. Sowohl Kant als auch Wittgenstein weisen das Fehlen eines Zusammenhangs zwischen Verstehen und Sich-Verstehen als folgenreiche Einschränkung aus - mit negativen Folgen nicht nur für Erkenntnis, sondern und vor allem

17 Vgl. u.a. zur Bedeutung von Perspektive und Perspektivität: Hartmut von Sass (Hg.): Perspektivismus. Neue Beiträge aus der Erkenntnistheorie, Hermeneutik und Ethik, Hamburg 2019.

18 Auf den Zusammenhang zwischen der Anerkennung von Perspektivität und dem Vermögen an Kippfiguren Alternativen zu erkennen, geht jüngst C. R. Sunstein ein: Cass R. Sunstein: Textualism and the Duck-Rabbit Illusion, 11 California Law Review, Online 463 (Nov. 2020), https://www.californialawreview.org/textualism-duck-rabbit-illusion, (letzter Zugriff September 2021).

19 Immanuel Kant: „Anthropologie in pragmatischer Hinsicht“, in: ders.: Werke in sechs Bänden, Bd. VI, Schriften zur Anthropologie, Geschichtsphilosophie, Politik und Pädagogik, Darmstadt 1998, 399-690, 411. 
auch für die Ethik und die allgemeine Anerkennung und Verwirklichung von Freiheit und Gleichheit aller Menschen. Beide Philosophen halten allerdings und aus praktisch-philosophischer Sicht bedeutungsrelevant fest, dass es Möglichkeiten der Übung gibt, gegen dieses Defizit anzugehen. Wittgenstein hält fest, dass das Vermögen, Aspektwechsel zu vollziehen, lernbar sei. Wer in einer reversiblen Figur nur die eine Alternative aktualisieren kann, wer also nur den Hasen, aber keine Ente zu sehen vermag, dem mag es helfen zu hören, dass die Ohren auch als Schnabel gesehen werden können etc. ${ }^{20}$ Voraussetzung dabei ist, dass jemand weiß, was ein Hase bzw. eine Ente ist und ausmacht. Reiz und Wirkung des Kipperlebnisses sind zwar nach Wittgenstein nicht zu delegieren, sie können aber, zumindest bei Personen, die nicht vollständig aspektblind sind, durch andere vorbereitet, vermittelt und gemeinsam geübt werden.

Kippfiguren erscheinen diesbezüglich als besonders geeignetes Übungsmaterial. Die Figuren strukturieren zwei (in seltenen Fällen drei oder mehr), eindeutig' aktualisierbare Varianten vor und laden dementsprechend den Betrachtenden gezielt dazu ein, das Kippen einzuleiten. Dass daran gleichsam das Vermögen geschult werden kann, an jeder Figur, jedem Begriff, jedem Bedeutungszusammenhang Kipp-Potentiale und also verdeckte Dimensionen, Relationen und Konstellationen auszumachen, ist nicht sicher. Die Fähigkeit, Kipp-Potentiale zu bemerken und das Kippen zuzulassen bzw. zu vollziehen, hat aber so grundlegende Bedeutung zum Beispiel eben auch für eine funktionstüchtige Demokratie, dass dieser Schulung und dem Vertrauen in diese Bildung hohe Priorität zukommen sollte. ${ }^{21}$ Es sind u. a. die Geisteswissenschaften, die Kipp-Potentiale nicht nur an Figuren, Bildern, Fotografien, Grafiken oder an Begriffen, Sätzen und Texten feststellen und untersuchen, sondern zudem dazu ermutigen und befähigen, Kipp-Momente auszuprobieren und emotional und intellektuell auszuhalten.

20 David Lauer: Anamorphotische Aspekte. Wittgenstein über Techniken des Sehens, in: Kyung-Ho Cha, Markus Rautzenberg (Hrsg.): Der entstellte Blick. Anamorphosen in Kunst, Literatur und Philosophie, München 2008, 230244 .

21 Zur Bedeutung des „Aspektwechsels“ für das individuelle Engagement für Demokratie: Aletta J. Norval: „Democratic Identification: A Wittgensteinian Approach." In: Political Theory 34.2, 2006, 229-255, hier: 232-238; Sara Fortuna: A un secondo sguardo. Mobile confine tra percezione e linguaggio, Rom 2002, 156-180. 
b) Gleichheit und Freiheit bilden zwei zentrale Prinzipien demokratischer Ordnungen. Wenn von Gleichheit als demokratischem Prinzip die Rede ist, dann ist dieses entsprechend durch ein unauflösbares Zusammenspiel mit dem Prinzip Freiheit gekennzeichnet. Demokratien gründen darum in einer paradoxalen Struktur: Wenn Menschen als gleiche und freie anerkannt werden, dann bedeutet dies auch, dass Menschen als gleiche und ungleiche anzuerkennen sind. ${ }^{22}$ Kippfiguren sind Gedankenexperimente, mit denen dieses Verständnis von der Gleichheit aller Menschen plausibel wird, denn sie führen vor, dass Verschiedenes Gleiches sein kann, auch wenn es sich der eigenen Wahrnehmung unmittelbar so nicht zeigt. Kippfiguren trainieren entscheidend das Differenzieren zwischen Wahrnehmen und Denken. Sie diktieren, dass etwas, das nicht unmittelbar gesehen werden kann, dennoch als schlüssig anzuerkennen ist. Niemand sieht zwar gleichzeitig die Linie als Linie und als Hase und als Ente. Es wäre aber unsinnig abzustreiten, dass Dasselbe tatsächlich Verschiedenes sein kann und Verschiedenes Dasselbe. Erleben-Denken oder kurz gesagt die Erfahrungen mit Kippfiguren fordern dazu auf, solche Denkschritte vorzunehmen. Die Analyse und Vertiefung des Denkerlebnisses macht deutlich, inwiefern Gleiches und Ungleiches miteinander einhergehen können. Kippfiguren vermitteln so die Einsicht in ein Problem, das sich der Begründung der Idee der Gleichheit aller Menschen stellt, jedenfalls dann, wenn die Prämisse geteilt wird, dass eine universale Gleichheit sinnlich nicht zwingend festzustellen ist und es auch nicht wünschenswert oder demokratisch wäre, Menschen so zu formen, dass diese ihre Individualität und Singularität verlieren würden. In der Wahrnehmung erscheinen Menschen zu Recht als Verschiedene. Auch rational ist das Prinzip demokratischer Gleichheit der Menschen nur unter Einbezug und Feststellung der Andersheit jeder einzelnen Person widerspruchsfrei zu begründen und einzufordern. Die Gleichheit der Menschen lässt sich nicht offensichtlich sehen, aber sie ist zu verstehen, und dazu laden die Kippfiguren nicht nur ein, sondern sie bieten hinreichenden Grund, ebendies anzuerkennen.

Das Spannungsverhältnis von Verschiedenheit und Gleichheit oder eben von Freiheit und Gleichheit lässt sich nicht auflösen, ohne dass damit demokratische Ordnungen in Gefahr kommen. Es wäre falsch, die Individualität politisch zu opfern und Menschen einander so anzugleichen, dass ihre 
Einzigartigkeit einer sichtbaren Egalität oder Uniformität weichte. Es wäre ebenso falsch, die Gleichheit abzuerkennen, nur weil sie nicht mit den Augen zu sehen ist. Demokratien als Ordnungen mit offenem Horizont sind darauf angewiesen, dass das Spannungsverhältnis, welches zwischen Freiheit und Gleichheit besteht, aufrecht erhalten bleibt und damit angewiesen auf Denkschritte, die das Gleiche und Verbindende von Ungleichem begreifbar machen.

Kippfiguren vermitteln auf spielerische und minimalistische und in vieler Hinsicht auch bescheidene Weise, was Jean-Jacques Rousseau in seiner Schrift über den Ursprung der Ungleichheit unter den Menschen ${ }^{23}$ ausführlich erläutert und anstrebt: Die Plausibilisierung der Gleichheit angesichts von Vielgestaltigkeit. Während das Gedankenexperiment des ersten Naturzustandes bei Rousseau die Herausbildung der Ungleichheit als Entfremdung darlegen muss, welche ,ihre Kraft und ihr Wachstum aus der Entwicklung unserer Fähigkeiten und den Fortschritten des menschlichen Geistes bezieht“ und „im Naturzustand fast gleich null ist“, ${ }^{24}$ widersetzen sich die Kippfiguren einer solchen Dichotomie-Setzung zwischen Natur und Kultur bzw. zwischen selbstvergessenem Erleben und selbst-bewusstem Denken. Rationalisierungsprozesse, so legen Kippfiguren dar, führen nicht zwingend weg von der Anerkennung der Gleichheitsidee wie es Rousseau beschreibt, sondern bezeugen und begründen im Gegenteil das enge Zusammenspiel von Erleben und Denken, wenn es darum geht, der Anerkennung von Gleichheit von Verschiedenem Relevanz zukommen zu lassen. ${ }^{25}$ Der $\mathrm{Zu}$ stand der Reflexion und Analyse ist, anders als es Rousseau auffasst, kein Zustand wider die Natur. ${ }^{26}$ Die rationalen Schritte, die durch das Erleben und Vollziehen des Aspektwechsels in Gang kommen, führen Menschen

23 Jean-Jacques Rousseau (1755): Diskurs über die Ungleichheit / Discours sur l'inégalité. Mit sämtlichen Fragm. und erg. Materialien nach den Orig.-Ausg. und den Hs. neu ed., übers. und komm. von Heinrich Meier. Kritische Ausg. des integralen Textes, Paderborn 2001.

24 Rousseau (1755): 61.

25 Kippfiguren umgehen damit das Problem, dass sich Rousseau stellt, wenn er die gleiche Natur aller Menschen zum Ausgangspunkt der politischen Idee der Gleichheit macht. Vgl. zu dieser Problematik bei Rousseau: Patricia Purtschert: Jenseits des Naturzustandes. Postkoloniale Lektüre von Hobbes und Rousseau, in: DZPhil, 60 (2012) 6, 861-882. 
nicht weg davon, das Prinzip von Gleichheit gegenseitig anzuerkennen, sondern im Gegenteil, so legt das Gedankenexperiment der Kippfiguren nahe, darauf hin. Erleben-Denken, also gedankliche Vertiefung, Bewusstwerdung und Rationalisierung des Vollzogenen sind unverzichtbar für die Begründung und Überzeugungskraft der menschenrechtlich verbürgten Gleichheit aller Menschen und ihrer grundrechtlichen Durchsetzung im Kontext demokratischer Ordnungen. ${ }^{27}$

\section{Paradoxien denken und Verbindendes vergegenwärtigen}

Das Gedankenexperiment der Kippfiguren führt zu zwei grundlegenden und für Demokratien elementaren Vermögen: Es macht verständlich, was zunächst als irritierender Widerspruch erscheint und es legt Ähnlichkeiten offen, wo zunächst allenfalls keine bemerkt wurden. Paradoxien denken und Verbindendes vergegenwärtigen können, sind Vermögen, die von Kippfiguren gleichzeitig geübt und in ihrer Bedeutung bestätigt werden. Auf beide Dimensionen soll hier abschließend noch einmal eingegangen werden. Dabei wird auch deutlich, dass Kippfiguren ihrerseits dafür sensibilisieren, dass sich die spezifische Wirkkraft von Gedankenexperimenten darin begründet, dass in ihnen Formen von Erleben-Denken ins Recht gesetzt werden.

Die Einladung der Kippfiguren zur Konfrontation mit gleichberechtigten Alternativen setzt, so wurde bereits ausgeführt, ein nachhaltiges Fragen in Gang. Das Erleben-Denken, das an und mit etwa dem Enten-Hase-Kopf mittelbar wird, reduziert zwar die Komplexität auf eine überschaubare Dimension und bereitet doch darauf vor, Personen als Andere und Freie und als Gleiche anzuerkennen und sie politisch auch so ins Recht zu setzen. Voraussetzung dafür ist die Fähigkeit, in Paradoxien denken zu können. ${ }^{28}$ Menschen sind verschieden und exakt besehen unvergleichbar und gleichzeitig

27 Auf die Bedeutung von Vermittlungsmedien für politische Bildung und gelingende Demokratie weisen auch etwa Friedrichs und Lange hin. Werner Friedrichs, Dirk Lange: Bewusstlose Demokratie? Das Bürgerbewusstsein in der (post-) demokratischen Konstellation der Gegenwart, in: Mörschel T., Krell C. (Hrsg.): Demokratie in Deutschland, Wiesbaden 2012, 53-70.

28 Kippfiguren ermöglichen zwar auch, in Ambivalenzen zu denken, aber vor allem üben sie den Umgang mit Paradoxien ein. Vgl. die Differenzierung zwischen Kippfiguren und Ambivalenz bzw. Paradoxie in: Einleitung, in: Kay Junge; Werner Binder; Kim-Claude Meyer; Mario Gerster (Hg.): Kippfiguren. Ambivalenz in Bewegung, Velbrück 2013. 
sind sie gleich, das heißt, tatsächlich vergleichbar in vielerlei Hinsicht. Das Prinzip demokratischer Gleichheit gründet in dieser Spannung, welche nicht aufzulösen ist, solange das Prinzip wirksam sein soll. Denn demokratische Gleichheit ist nicht $\mathrm{zu}$ verwechseln mit Vorstellungen von Egalitarismus resp. sozialer Gleichmachung aller und auch nicht mit Vorstellungen von radikalem Individualismus oder extremen Formen des Multikulturalismus. Während im ersten Fall die Vielgestaltigkeit menschlicher Lebensformen reduziert wird, um Gleichheit sichtbar durchzusetzen, werden im zweiten Fall die Besonderheiten von Personen und/oder Gruppen soweit ausdifferenziert und hervorgehoben, dass darüber das Gleiche abgeschwächt oder völlig ausgeblendet wird. Das Prinzip demokratischer Gleichheit hingegen gründet in einer paradoxalen Struktur, die sich daraus ergibt, dass nicht auf die eine noch auf die andere Seite geschwenkt wird, sondern dass das Sowohl-alsauch bekräftigt bleibt von Verschiedenheit und Gleichheit, von Unvergleichbarkeit und Verbundenheit aller Menschen.

Nicht nur wird durch die Kippfiguren deutlich, dass Verschiedenes und Gleiches plausibel zusammen zu denken sind, obwohl dies zuerst als ein irriger Widerspruch erschienen ist, sondern darüber hinaus offenbaren Kippfiguren auch, dass allen Personen vergleichbare Wahrnehmungsprozesse und Erkenntnisschritte eignen. Nicht nur das eigene Sehen ist als eine Form von Deuten zu erkennen, sondern auch die Wahrnehmung anderer Personen ist als Perspektive zu erkennen. Kippfiguren vermitteln Erfahrungen und legen zudem und gleichzeitig offen, dass und wie Menschen Erfahrungen machen (können). Die dabei sich einstellenden Prozesse verweisen auf verbindende Eigenschaften aller Menschen.

Kippfiguren verweisen auf mehrere Arten auf Verbindendes von Menschen und belegen es. Ähnliche Abläufe im Umgang mit Kippfiguren sind jedenfalls bei vielen Menschen auszumachen: Zunächst ist festzustellen, dass sich Kippfiguren allen zuerst in einer Konkretisierung zeigen. Sodann ist festzuhalten, dass es vielen bald einmal möglich ist, zwei (oder mehrere) Konkretisierungen zu realisieren. Die Überraschung darüber, noch etwas zu sehen, stellt sich ebenfalls bei vielen ein und macht einen allgemeinen Reiz dieser Experimente aus. Wie dargelegt wurde, legt das Sehen weiterer Visualisierungen an Kippfiguren darüber hinaus etwas an einem selbst offen. Das Bemerken des eigenen Sehens als eine Perspektive ermöglicht einen elementaren Erkenntniszugewinn und zwar nicht nur in Bezug auf sich selbst, sondern gleichzeitig auch auf die anderen und auf die Relation zwischen sich und anderen. Perspektivität als allgemeine Eigenschaft von Wahrnehmun- 
gen wird im Verlauf der Reflektion des Erlebens, der Wiederholung des Vorgangs und der damit verbundenen gedanklichen Vertiefung sinnfällig.

Während die bisher genannten Dimensionen im Umgang mit Kippfiguren auf Ähnlichkeiten aller Menschen hinweisen, gibt es zudem Unmöglichkeiten, welche das Gleichheitsprinzip weiter bekräftigen. So ist interessant, dass niemand vermag, beide (oder alle) möglichen Konkretisierungen gleichzeitig zu aktualisieren, und es niemandem gelingt, den Wechsel selbst zwischen den beiden (oder mehreren) Alternativen in den Blick zu nehmen.

Während des Hin- und Her-Kippens zwischen den möglichen Konkretisierungen von Kippfiguren wird gewahr, dass sich im Moment stets je nur etwas profilieren und sehen lässt und sich gleichzeitig je etwas entzieht. Selektivität und Temporalität des eigenen Zugriffs werden so offensichtlich und davon ausgehend und darüber hinaus als allgemeine Eigenschaft von Wahrnehmung plausibel ableitbar. Die allgemeinen Eigenschaften bewussten Zugriffs, selektiv und nachträglich zu sein, werden auch daran deutlich, dass das Kippen zwar angestrebt, vollzogen und erlebt werden kann, es aber niemandem gelingt, das Kippen selbst, also den Moment zwischen den Zuständen, in den Blick zu nehmen. Die Aktualisierung der Ente bzw. des Hasen ist bedingt durch einen Vorgang, der sich selbst eines direkten, sinnlichen Zugriffs widersetzt. Das Ereignis des Aspektwechsels kann nur angenähert werden durch eine Art von Rekonstruktion und Beschreibung bzw. durch Abstraktion und Rationalität. Weil man das Kippen vollzieht und den Wechsel erlebt und diesen als Voraussetzung des Kippspiels und seiner Einsichten nicht sinnvoll negieren kann, rückt der Aspektwechsel zudem als Angelpunkt von Erleben und Denken in den Fokus. Obwohl Ente und Hase nie gleichzeitig aufleuchten können und das Kippen, also der Moment, in dem das Aufleuchten sich ereignet, nicht festgehalten werden kann, schärft sich im Erleben-Denken, das durch den Kippmoment initiiert wird, gerade nicht vor allem der Sinn für die sinnlich wahrgenommene Relation des Entweder-Oder, sondern und besonders auch für das abstraktere Sowohl-alsauch.

Während im Bemerken der Entweder-Oder-Relation das Bewusstsein geschärft wird für das, was sich dem Zugriff stets entzieht, also für das Ausgeblendete oder Übersehene, für die Perspektivität des Zugriffs, wird in der rationalen Vergegenwärtigung des Wahrnehmbaren und im daraus resultierenden Begreifen der Sowohl-als-auch-Relation das Bewusstsein sensibilisiert einerseits für das allgemein Defizitäre und Bedingte von Wahrnehmungen und andererseits für die Möglichkeit, dass dasselbe sich als dasselbe 
und als nicht dasselbe herausstellen kann. Perspektivität der Zugriffe und Gleichheit von als verschieden Wahrgenommenem sind Erkenntnisse, die an und mit Kippfiguren nicht sinnlich, sondern nur und erst im Zusammenspiel mit Reflektion und rationaler Schlussfolgerung verständlich werden. Nicht zuletzt votieren Kippfiguren entsprechend performativ und also zeigend und sagend für einen gleichberechtigten Einbezug von Erleben und Denken zugunsten der Plausibilisierung u. a. des Prinzips demokratischer Gleichheit. 